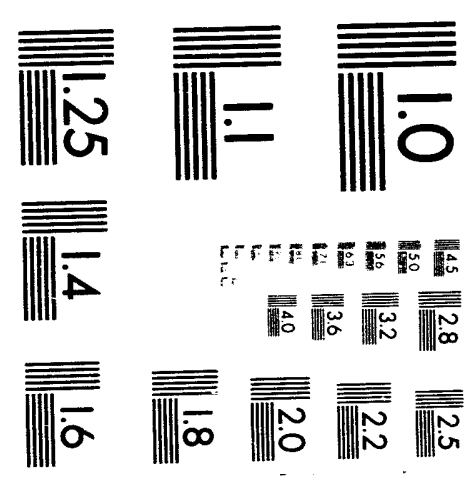



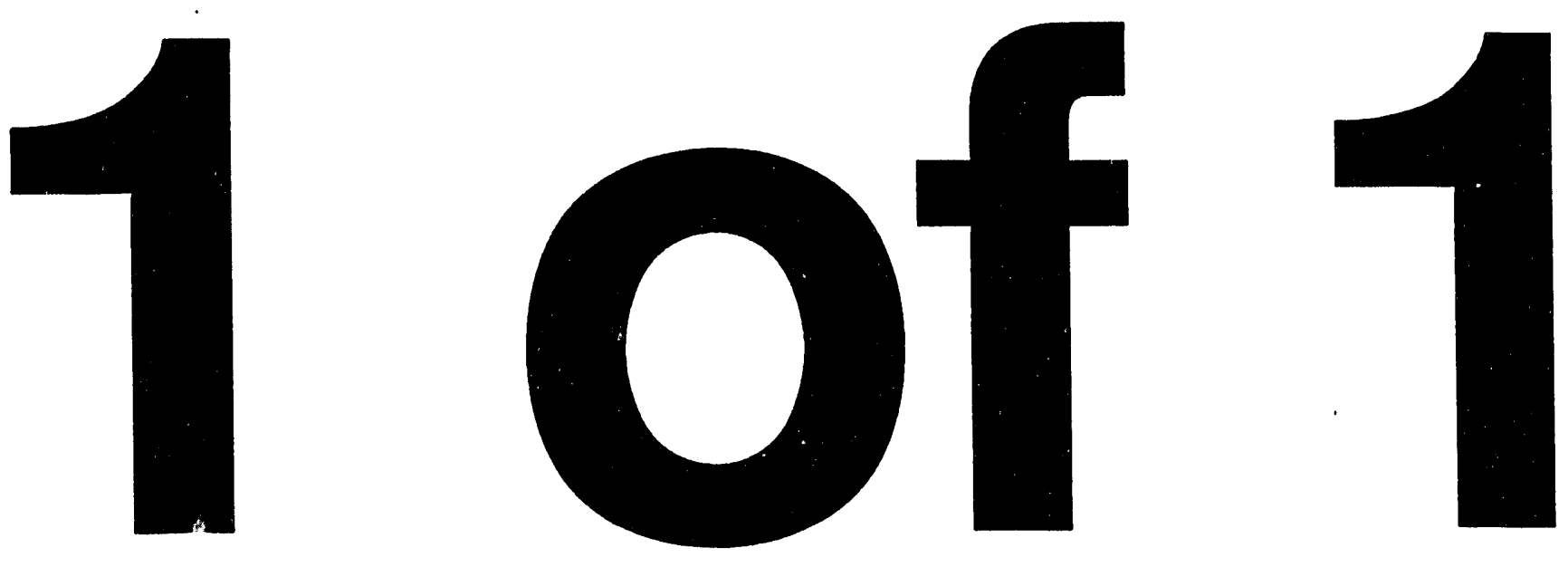


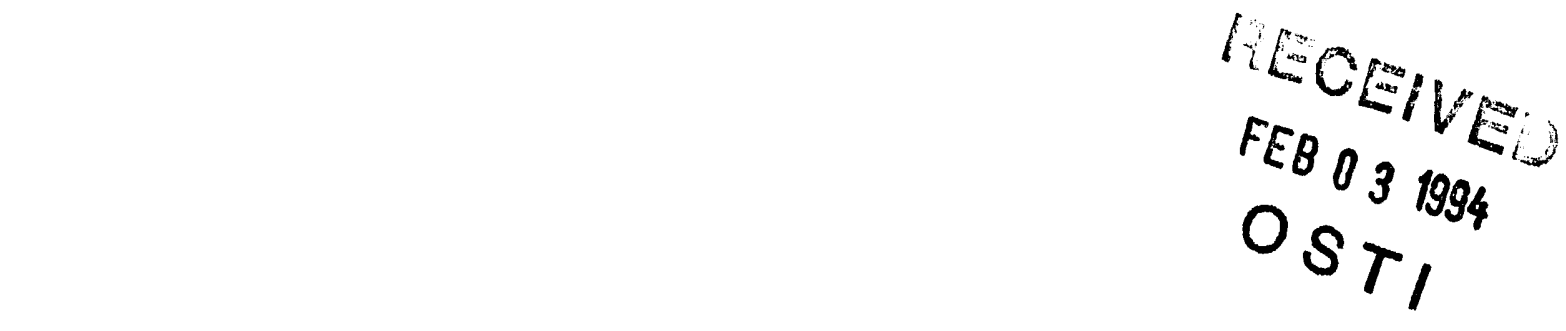

\title{
High Resolution Three Dimensional Microscopy of Biological Microstructures Using Zone Plate Lenses with X-Ray Laser Illumination
}

\author{
Jim Trebes
}

December 12, 1990

This is an informal report intended primarily for internal or limited external distribution. The opinions and conclusions stated are those of the author and may or may not be those of the Laboratory.

Work performed under the auspices of the U.S. Department of Energy by the Lawrence Livermore National Laboratory under Contract W-7405-Eng-48. 


\section{DISCLAIMER}

This document was prepared as an account of work sponsored by an agency of the United States Government. Neither the United States Government nor the University of California nor any of their employees, makes any warranty, express or implied, or assumes any legal liability or responsibility for the accuracy, completeness, or usefulness of any information, apparatus, product, or process disclosed, or represents that its use would not infringe privately owned rights. Reference herein to any specific commercial products, process, or service by trade name, trademark, manufacturer, or otherwise, does not necessarily constitute or imply its endorsement, recommendation, or favoring by the United States Government or the University of California. The views and opinions of authors expressed herein do not necessarily state or reflect those of the United States Government or the University of California, and shall not be used for advertising or product endorsement purposes.

This report has been reproduced directly from the best available copy.

A vailable to DOE and DOE contractors from the Office of Scientific and Technical Information P.O. Box 62, Oak Ridge, TN 37831

Prices available from (615) 576.8401, FTS 626-8401

Available to the public from the National Technical Information Service

US. Department of Commerce 5285 Port Royal Rd. Springfield, VA 22161 


\section{High Resolution Three Dimensional Microscopy of Biological}

Microstructures Using Zone Plate Lenses with X-ray Laser Illumination*

One of the goals of biomedical research is the development of imaging techniques capable of producing high resolution ( $300 \AA)$ three dimensional images of structures within live cells. Recent developments in zone plate lenses at LBL and in $x$-ray lasers at LLNL indicate that flash three dimensional $x$-ray microscopy of live biological objects can be achieved in the near term. This concept ofr a microscope utilizes an $x$-ray laser to backlit immunogold labeled biological objects. These backlit objects are then imaged with low f-number, high resolution zone plate lenses. Backlighting and imaging along several different directions simultaneously allows a three dimensional image to be obtained using tomographic techniques.

\section{Two Dimensional Imaging}

The best way to investigate the possibilities of this new high resolution microscope concept is to quantitatively examine its use with a realistic biological objects. The object to be imaged will be a cell 10 microns in diameter and 1 micron thick. It will be mounted, dry, on a $500 \AA$ thick boron nitride membrane in vacuum. Imaging a wet cell in an environmental chamber will be considered later in this paper. The actin fibers within the cell will be labeled with $500 \AA$ gold microspheres using immunogold labeling. A single view system capable of good two dimensional resolution will be analyzed first. A schematic of this system is shown in figure 1. A multiple view system capable of good three dimensional resolution will considered second. The Ni-like Ta laser operating at $44.83 \AA$ will be used in both systems. The beam is focused with a demagnification of 6 onto the cell using spherical multi-layer mirrors. The spherical mirror is operated at near normal incidence to minimize abberrations in the focal spot. The $x$-rays scattered by the gold spheres are imaged with zone plates onto $x$-ray film detectors. The image magnification is $\sim 200$. The film is protected from UV and visible light with a thin filter. 


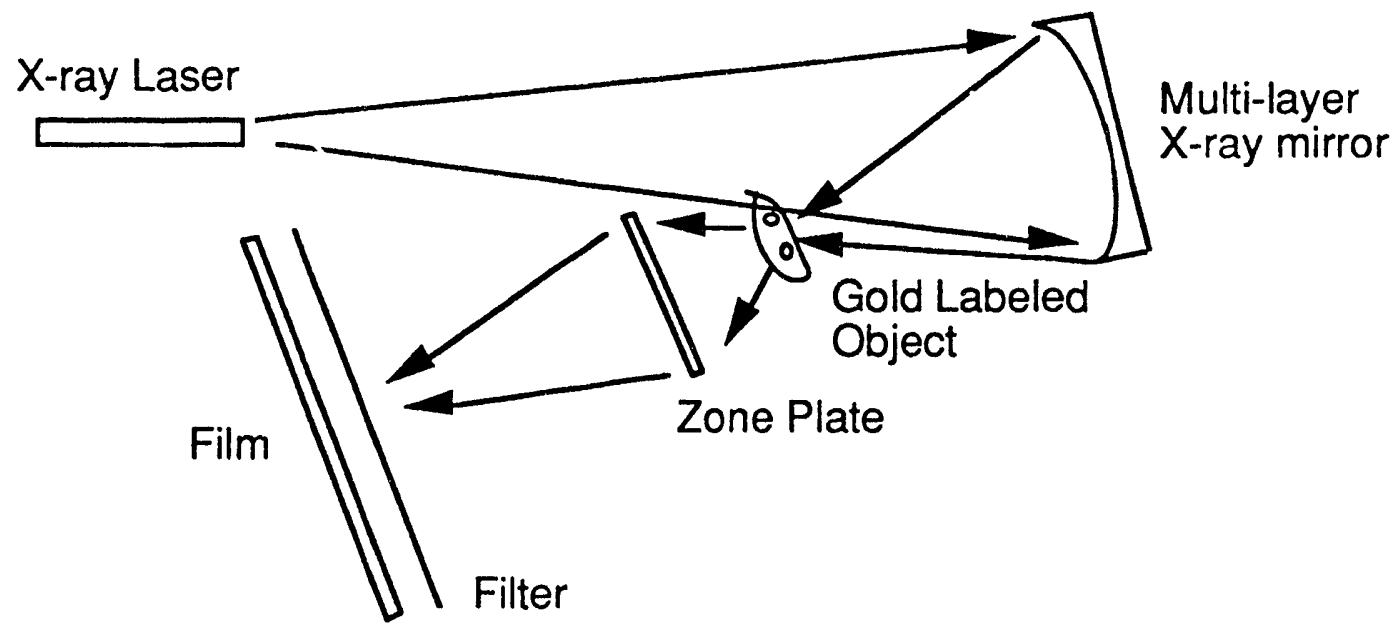

Figure 1. Schematic of zone plate -x-ray laser microscope capable of producing high resolution two dimensional images of gold labeled biological microstructures.

Knowing the sizes of components of the system and the calculated $x$-ray responses allows the required $x$-ray laser energy to be calculated. The number of detected photons in the image is given by:

$$
N_{\text {det }}=\mathrm{FT}_{\text {sub }} T_{\text {protein }} T_{\text {filter }} \varepsilon_{\text {film }} \varepsilon_{\text {z.p. }}{ }_{\text {fo }}
$$

where $F=x$-ray laser fluence at object

$T_{\text {sub }}=$ substrate transmission

Tprotein = protein transmission

$T_{\text {filter }}=$ filter transmission

$\varepsilon_{\mathrm{film}}=\mathrm{x}$-ray film detective quantum efficiency

E.p. $\quad=$ zone plate efficiency

zone plate

$f=$ fraction of scattered $x$-rays collected by

$\sigma \quad=$ total elastic scattering cross-section.

\section{Elastic Scattering Cross-section $\sigma$}

The gold labled cell is assumed to produce elastic scattering only from the spherical gold labels. Furthermore the cell is assumed to be uniform over scalelengths large compared to the gold sphere diameter and is treated as an absorbing filter in the signal level calculations. The scattering from the gold is 
calculated using the anomalous diffraction model. This model is valid when $2 \pi a / \lambda \gg 1$ and $(4 \pi a / \lambda) *(n-1) \sim 1$, where $a$ is the sphere radius and $n$ is the index of refraction. For small gold spheres and at soft $x$-ray wavelengths these conditions are true. Using this model the elastic scattering cross-section can be calculated as an efficiency factor $Q$ times the geometric cross-section $\pi r^{2}$. The value of $Q$ for several gold sphere diameters and at a wavelength of $45 \AA$ is shown in Table 1 . The index of refraction of the gold is taken to be that calculated by London: $n=1-7.4 \times 10^{-3}$-i $1.03 \times 10^{-}$ 2. The index of the surrounding cell is ignored since its value is small compared to that of gold.

\begin{tabular}{cc} 
Diameter in $\AA$ & $\begin{array}{c}\text { Table } \\
\mathrm{Q}\end{array}$ \\
\hline 300 & \\
500 & .11 \\
750 & .22 \\
1000 & .41 \\
1500 & .54 \\
2000 & .76 \\
& .89
\end{tabular}

At small diameters such as $300 \AA$ the Rayleigh-Gans scattering approximation is valid and the scattering cross-section is sma!!" compared to the geometric cross-section. The object is optically thin in this case. At large diameters such as $2000 \AA$ the object is opaque and the cross-section is comparable to the geometric cross-section. The total elatic scattering cross-section for a $500 \AA$ diameter gold sphere is $4.3 \times 10^{-4} \mu^{2}$.

\section{Detected Fraction $f$}

The fraction of scattered $x$-rays detected is a function beam stop size, zone plate size, and gold label size. A beam stop is used to eliminate the zero order component from the zone plate image. Its effect is included in the zone plate efficiency. Using LBL $f / 6.7$ zone plates with $500 \AA$ gold spheres results in $\mathrm{f}=0.8$.

\section{Film Response}

Kodak 101 film has a film density of 0.5 for an exposure of 1 photon/micron 2 . The fog level is $\sim 0.1$ in density. With this level of exposure and fog level single photon exposure can be measured with the PDS film densitometer. The quantum efficiency of exposing a single film grain with a $277 \mathrm{ev}$ photon is $\sim 100 \%$. This 
results in a detected quantum efficiency of $\sim 50 \%$. The detected quantum efficiency is defined as the ratio of the signal-to-noise ratio in the incident $x$-rays to the signal-to-noise ratio in the recorded image.

\section{Zone Plate Efficiency}

Based on discussions with Dave Attwood of LBL the expected zone plate efficiency is approximately $5 \%$. This $5 \%$ assumes a beam stop in the center of the zone plate which is at least 10 microns in radius. The existing $L B L$ zone plates have a diameter of 35 microns and a focal length for $45 \AA$ x-rays of 221 microns. A beam stop may be required to stop the undiffracted portion of the $x$-ray illumination beam. Minimzing the size of this stop will probably require the use of a limiting aperture at the object. Further discussions are required with LBL personnel to clarify this issue. Higher efficiencies may be available in the near future. In any case alignment will be quite difficult.

\section{Filter Transmission}

A filter is included to prevent exposure of the film to visible and ultraviolet light from the $x$-ray laser target. The filter will likely consist of $1000 \AA$ thick Lexan and $2000 \AA$ thick titanium. The mean free path at $45 \AA$ for Lexan is 2.7 microns giving a transmission of $96 \%$. The mean free path in titanium is 0.27 micons giving a transmission of $48 \%$ for a net transmission of $46 \%$. A transmission of $61 \%$ could be obtained if Luxel can coat scandium. The visible light transmission of $\mathrm{Ti}$ is extremely low so that $1000 \AA$ thick coatings could be used if pinhole free filters could be made, then the transmission would be $67 \%$.

\section{Protein Transmission}

The cell is modeled as pure DNA 1.0 microns thick. The mean free path at $45 \AA$ based on London's calculations is $7000 \AA$. This gives a transmission of $24 \%$. This is a pessimistic attenuation since some of the cell is composed of water and protein which have a smaller $x$-ray attenuation.

\section{Substrate Transmission}

The substrate consists of $500 \AA$ of boron nitride whose mean free path at $45 \AA$ is $2400 \AA$. The transmission of the substrate is $81 \%$.

Net Result 
Multiplying the reponses of the various components together results in the following expression for the number of detected photons:

$$
N_{\text {det }}=7.7 \times 10^{-7} \mathrm{~F}
$$

where $F$ is the $x$-ray flux in photons $/ \mu^{2}$ incident on the cell. Approximately 100 photons/gold label must be detected for good signal-to-noise in the image. This results in the required flux on

the sample being $1.3 \times 10^{8}$ photons $/ \mu^{2}$ for a normal incidence beam.

\section{Required X-ray Laser Output}

The required flux at the sample is achieved by relaying and focusing the $x$-ray laser beam on the sample with a multilayer mirror. The reflectivity is assumed to be $10 \%$. Higher reflectivities may be available in the near future. The demagnification is assumed to be 6 . This results in a 20 micron spot at the sample plane using the estimated source size of the $x$-ray laser of 120 microns in diameter. Including the effects of the mirror and the demagnification, the required flux at the $x$-ray laser is $3.6 \times 10^{7}$ photons $/ \mu^{2}$. Currently the $x$-ray laser has an output flux of $6 \times 10^{7}$ photons $/ \mu^{2}$ at a wavelength of $44.83 \AA$. The current Ta $x$-ray laser has sufficient output energy to produce $500 \AA$ resolution two diemsional images of gold labeled dry cells.

Producing images of wet cells will require additional output. A wet cell holder will need to have $\sim 1000 \AA$ thick windows to maintain atmospheric pressure. This forces an increase by a factor of 2 due to the added attenuation of the thicker windows. The holder will also contain approximately 1 micron of water forcing an increase of an additional factor of 3 due to its absorbtion. Increasing the resolution from $500 \AA$ to $300 \AA$ can be accomplished by using smaller gold labels. This will require increasing the output energy by another factor of 11 due to the reduced crosssection and the smaller fill factor of the zone plate which result from using $300 \AA$ diameter gold labels. The net effect is that to produce $300 \AA$ resolution, two dimensional images of wet, gold labeled cells will require increasing the existing Ta $x$-ray laser 
output energy by a factor of 40 . This can be accomplished using a double pass geometry.

\section{Three Dimensional Imaging}

Three dimensional images of gold labeled biological microstructures can be produced using a multi-view system similar to that proposed for $x$-ray laser holography. Essentially several (3-4) images are obtained along different lines of sight through the object. The relative angle between these lines of sight need to be at approximately 90 degrees with respect to each other. The resulting images are added tomographically to produce a composite image with a three dimensional spatial resolution comparable to the two dimensional resolution obtained from a single view. A schematic of a conceptual system is shown in figure 2. The $x$-ray laser beam is split into three parts by a segmented multi-layer $x$-ray mirror. Each part is focused onto the object along independent directions. The demagnification of the mirror is a factor of 6 . Each direction has its own detector.

The required $x$-ray laser energy output is increased by the inclusion of the segmented mirror. For a $10 \%$ reflectivity, three segment mirror an additional factor of 30 in $x$-ray laser output energy is needed. Since the focused $x$-ray laser beams are now incident on the object at approximately 45 degrees another factor of 1.4 is required to compensate for the reduced fluence due to the angle. The net result of this is that $500 \AA$ resolution, three dimensional imaging of dry objects will require increasing the current Ta $x$-ray laser output by a factor of 25 . Three dimensional imaging of wet objects with $300 \AA$ resolution will require increasing the existing $T a x$-ray laser output by a factor of 1660 . The improvements in $x$-ray laser performance required for several microscope resolution capabilities are summarized in Table 1. 


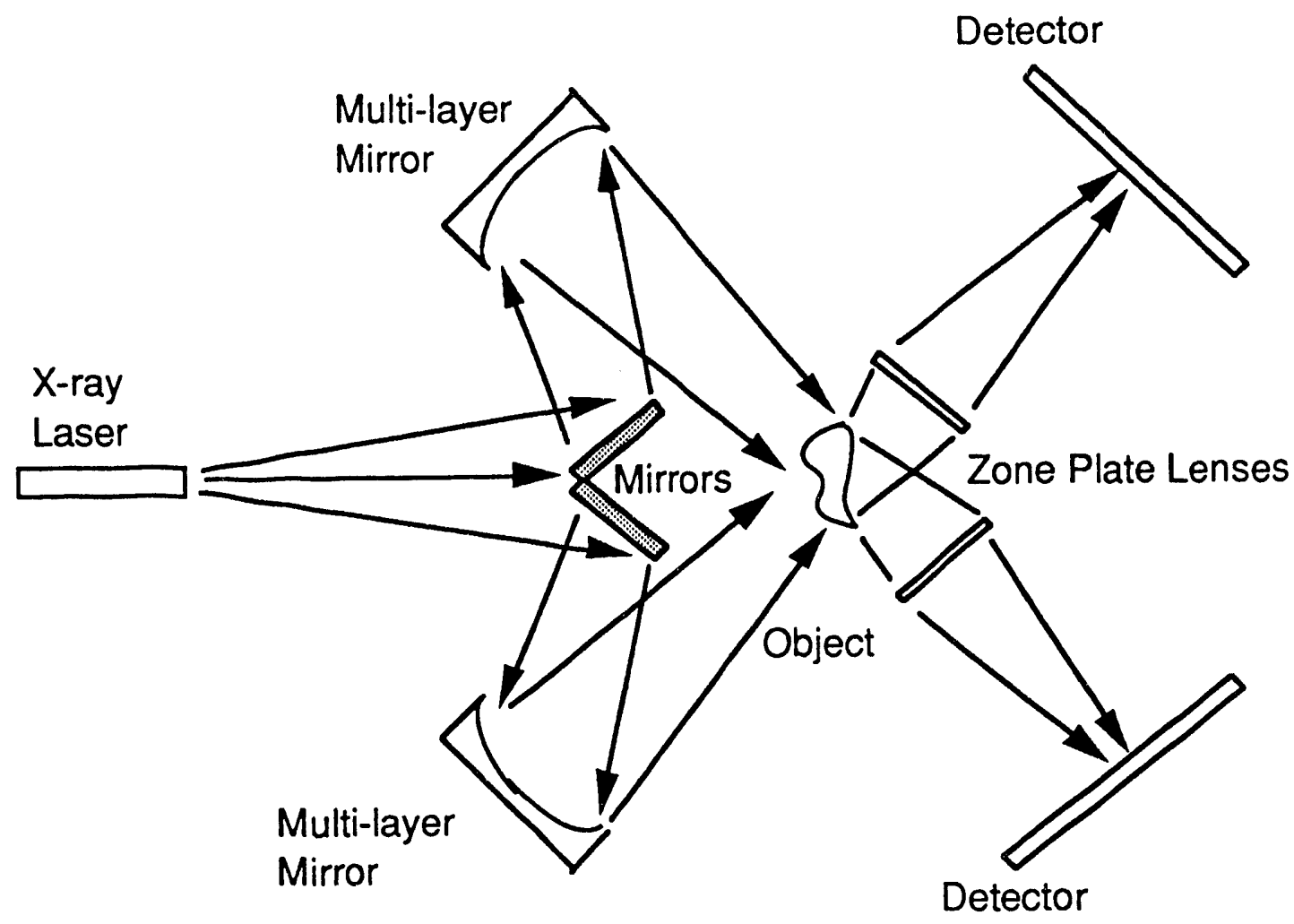

Figure 1. Schematic arrangement of $x$-ray laser zone plate microscope utilizing multiple views to obtain good depth resolution. Only 2 beamlines are shown.

\section{required}

\section{Table 1 \\ spatial resolution $x-r d y$ laser gain}

$\begin{array}{lr}500 \AA, 2-D, \text { dry } & \text { none } \\ 500 \AA, 2-D, \text { wet } & 3.6 \\ 500 \AA, 3-D, \text { dry } & 25 \\ 500 \AA, 3-D, \text { wet } & 150 \\ 300 \AA, 2-D, \text { dry } & 6.6 \\ 300 \AA, 2-D, \text { wet } & 40 \\ 300 \AA, 3-D, \text { dry } & 277\end{array}$


Table 1. Shown is the factor of increase in $x$-ray laser output energy required to obtain various levels of resolution with the object being either wet or dry.

\section{Conclusion}

It should be possible in the near future to use existing $x$-ray laser and zone plate technology to build a multiple line of sight, high three dimensional resolution $x$-ray microsocpe for gold labeled biological objects. Construction of a microscope with a $500 \AA$ three dimensional resolution of dry objects or a microsope with a $300 \AA$ two dimensional resolution of wet objects appears to be feasible now. The major issues appear to be:

1) the size of the beam stop for the zone plate and its effect on the efficiency ( it may be possible to operate without a beam stop),

2) off-axis abberrations may severely limit the obtainable resolution with large biological objects (these can be minimized by post processing of the images with a priori information such as the known size of the gold microsphere labels), and

3) alignment (maybe the most difficult problem).

It should be noted that at the highest levels of resolution the abberations inherent in single zone plate lenses and the difficulty of producing $\sim 1600$ increases in output energy may force the use of holography to obtain 3-D images. Note that increasing the output energy by a factor of 1600 is equivalent to increasing the spatial coherence by a factor of 40 when using holographic imaging. Due to limitations imposed by gain saturation effects, it may be ultimately easier to achieve high resolution imaging with holographic techniques. 

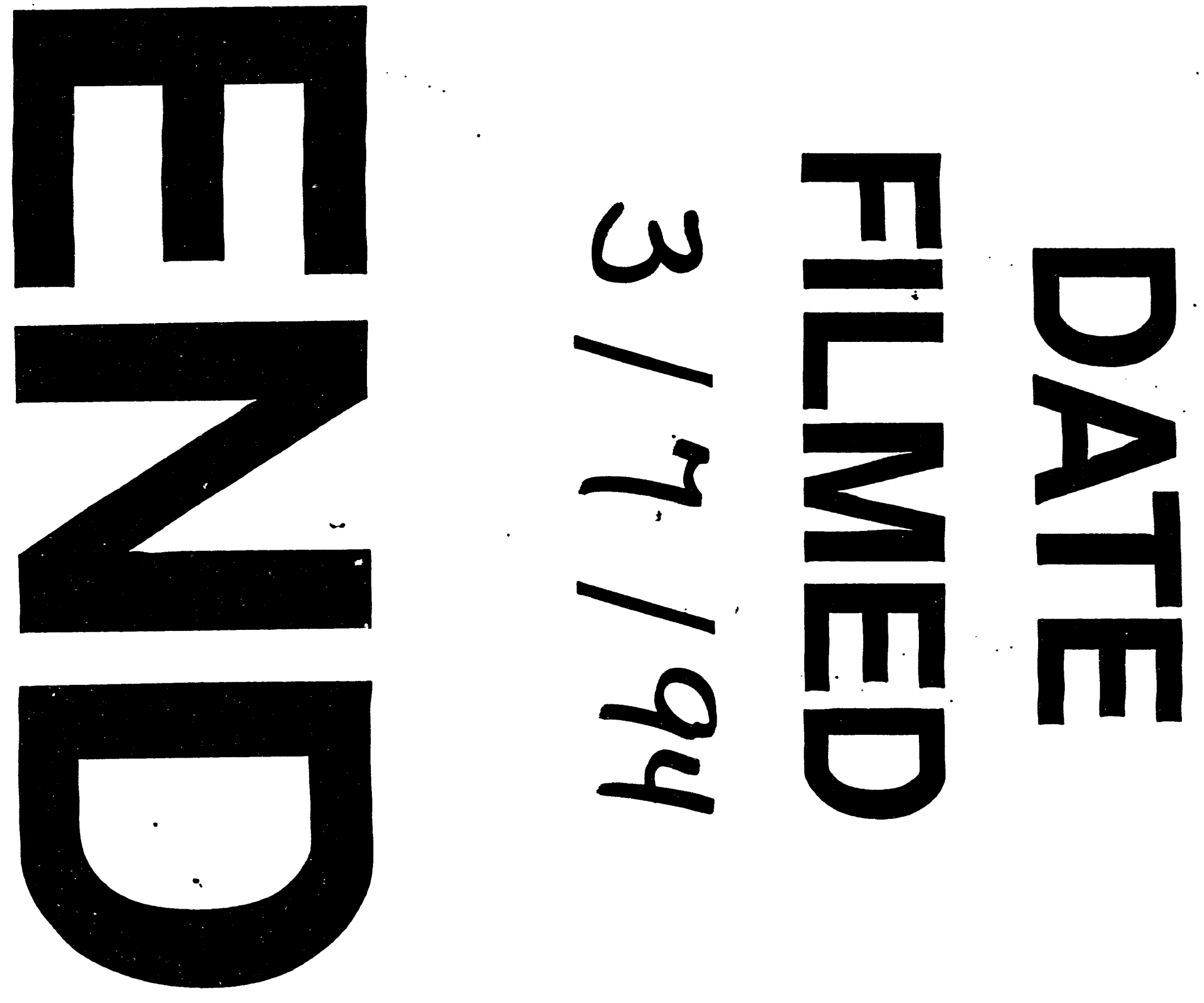